\title{
Hemobilia y hemocolecisto agudo como forma de presentación inusual del cáncer de vesícula biliar
}

\author{
Hemobilia and acute hemocolecyst as an unusual presentation of gallbladder cancer \\ José J. Motos-Micó*, Javier Cortés-Climent, Lirios Ferri-Candela, Francisco Arlandis-Félix y \\ Carlos Serra-Díaz \\ Servicio de Cirugía General y del Aparato Digestivo, Hospital Virgen de los Lirios, Alcoy, Alicante, España
}

\begin{abstract}
Resumen
El cáncer de vesícula biliar que se presenta como una colecistitis aguda asociada a hemocolecisto y hemobilia es muy infrecuente. Hasta la fecha hay pocos casos informados en la literatura. Presentamos un caso de carcinoma de vesícula biliar diagnosticado tras colecistectomía de urgencia, realizada por hemobilia y colecistitis aguda por hemocolecisto.
\end{abstract}

Palabras clave: Hemobilia. Hemocolecisto. Vesícula biliar.

\begin{abstract}
Gallbladder cancer that presents as acute cholecystitis associated with hemocholecyst and hemobilia is a rare entity. There are few cases reported in the literature. We present a case of gallbladder carcinoma diagnosed after emergency cholecystectomy, performed due to hemobilia and acute cholecystitis due to hemocholecyst.
\end{abstract}

Key words: Hemobilia. Hemocholecyst. Gallbladder.

\section{Introducción}

El cáncer de vesícula biliar que se presenta como una colecistitis aguda asociada a hemocolecisto y a hemobilia es muy infrecuente. Hasta la fecha, hay muy pocos casos informados en la literatura.

\section{Caso clínico}

Varón de 66 años con antecedentes de diabetes mellitus tipo 2 y artritis reumatoide que acude a urgencias por ictericia y dolor abdominal en el cuadrante superior derecho durante las últimas 24 horas, asociado a náuseas y vómitos. La exploración física revela un signo de Murphy dudoso. Los datos de laboratorio muestran bilirrubina total de $6.14 \mathrm{mg} / \mathrm{dl} \mathrm{y}$ aumento leve de las enzimas hepáticas. Durante su ingreso, el paciente evoluciona de manera tórpida, presentando elevación de los reactantes de fase aguda, la amilasa y la lipasa. Se realiza tomografía computarizada urgente, compatible con colecistitis aguda complicada con signos de sangrado intravesicular sin poder descartar la presencia de una lesión sólida intravesicular.

\footnotetext{
Correspondencia:

*José J. Motos-Micó 
Se decide intervención urgente, presentando colecistitis aguda complicada con hemocolecisto y hemobilia. Se realizan colecistectomía, coledocotomía, coledocoscopia y colocación de Kehr. La anatomía patológica revela un adenocarcinoma de vesícula biliar tipo indiferenciado pleomórfico que infiltra la capa muscular (T1bNxMx), sin poder asegurarla por extensa autólisis por defecto de fijación en la pieza quirúrgica.

El paciente se somete a una segunda intervención con bisegmentectomía hepática IVB y V, además de disección de los ganglios linfáticos del hilio hepático, sin evidencia de infiltración neoplásica.

A los 3 meses, el paciente reingresa por fiebre posquirúrgica y en la tomografía computarizada abdominal se objetivan varias imágenes sugestivas de metástasis hepáticas en los segmentos VIII, VII y VI, además de posibles implantes en la musculatura lateral derecha de la pared abdominal y el ligamento gastrohepático. La biopsia hepática informa de metástasis hepática de adenocarcinoma bien diferenciado.

El paciente recibe tres ciclos de quimioterapia de primera línea con cisplatino-gemcitabina en progresión de la enfermedad y fallece a los 9 meses de la primera intervención quirúrgica.

\section{Discusión}

El tumor de la vesícula biliar es la neoplasia maligna más común del tracto biliar, con una incidencia de 2.5 casos por 100,000 habitantes'.

El hemocolecisto ocurre en menos del $1 \%$ de los tumores de vesícula biliar y se define como la presencia de una hemorragia en el interior de la vesícula biliar, siendo descrito por Fitzpatrick por primera vez en $1961^{2}$. La presencia de sangre en el interior de la vesícula biliar puede ocurrir por varias razones: colecistitis aguda, traumatismo abdominal, biopsia hepática, neoplasias biliares, hemobilia, mucosa gastrointestinal heterotópica, aneurismas de arterias vecinas a la vesícula biliar, diátesis hemorrágica, parasitosis biliar e isquemia ${ }^{2-4}$.

El cáncer de vesícula biliar que se presenta como colecistitis aguda asociado con un hemocolecisto es infrecuente y hay pocos casos descritos en la literatura ${ }^{5}$. Suele manifestarse con síntomas inespecíficos, como dolor abdominal en el hipocondrio derecho y fiebre ${ }^{6,7}$.

Actualmente, los mejores estudios de imagen para diagnosticar estos tumores son la ecografía abdominal y la tomografía computarizada. La ecografía se ha

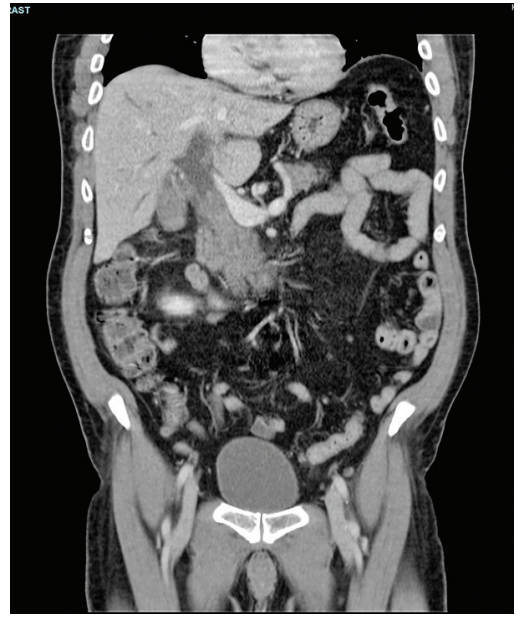

Figura 1. Colecistitis aguda complicada con hemocolecisto $y$ hemobilia.

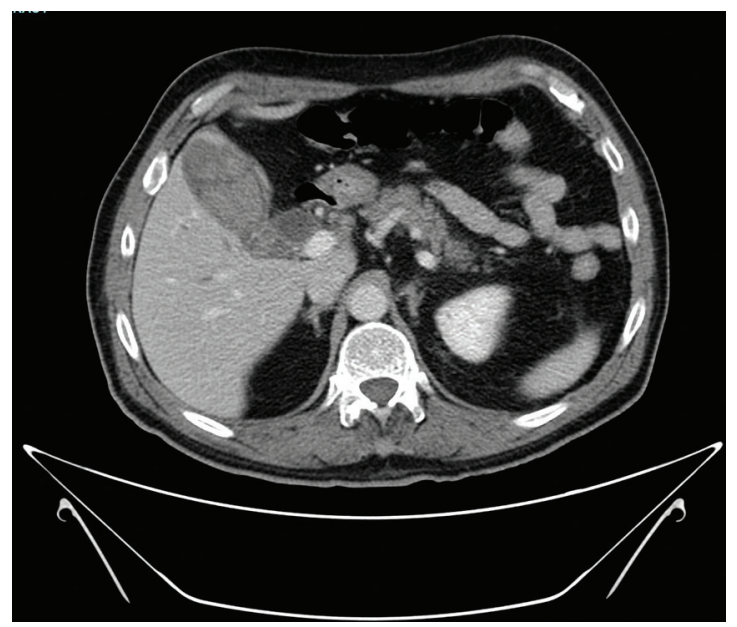

Figura 2. TAC abdomen en proyección axial: colecistitis aguda complicada con signos de sangrado intravesicular.

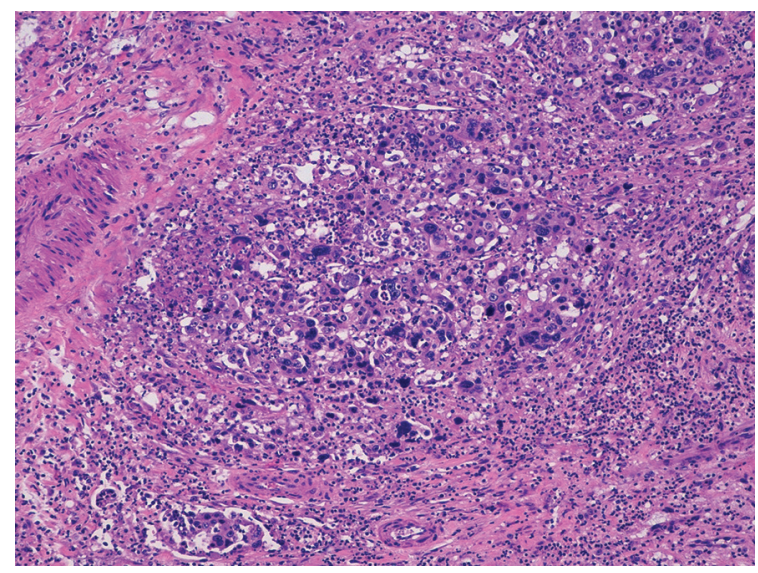

Figura 3. Imagen histológica con hematoxilina-eosina de adenocarcinoma de vesícula biliar compuesto por atipia celular muy intensa, incluyendo células gigantes de morfología sarcomatoide y escasa formación de luces glandulares. 
utilizado durante mucho tiempo en el diagnóstico de enfermedades agudas, como la colecistitis. Desafortunadamente, los pólipos y los carcinomas tienen una ecogenicidad similar a la de la pared de la vesícula biliar, lo que dificulta distinguirlos de una pared engrosada secundaria a cambios inflamatorios agudos. Dada la rareza del cáncer de vesícula biliar y la asociación aún más rara con un hemocolecisto, el diagnóstico rápido todavía elude al radiólogo y al cirujano ${ }^{5}$.

La resección completa del tumor proporciona la única posibilidad de cura, pero conseguir un diagnóstico preoperatorio temprano es esencial. De hecho, la tasa de supervivencia a 5 años después de la resección con intención curativa se ha descrito en menos del $17 \%$ de los casos $^{5}$. La quimioterapia adyuvante no ha demostrado su eficacia en el carcinoma de vesícula biliar, aunque la mayoría de los estudios estaban limitados por el pequeño número de casos incluidos $^{8}$. El agente quimioterápico más estudiado es el 5 -fluorouracilo, con una tasa de respuesta de solo un $10-24 \%$.

Un paciente con hemocolecisto puede presentar varios escenarios clínicos. La hemobilia se presenta clásicamente como dolor de cólico biliar, ictericia y hemorragia. Dependiendo de la cantidad y la tasa de sangrado, la sangre puede coagularse en varios lugares. Si la sangre no se coagula en el tracto biliar, puede ocurrir hematemesis o melena. Si la sangre se coagula dentro del conducto biliar, puede causar ictericia obstructiva o pancreatitis. El sangrado inicialmente produce coágulos intravesiculares que causan distensión vesicular y una clínica típica de colecistitis aguda. Si no se trata, puede producirse la perforación de la vesícula y originarse un abdomen agudo con hemoperitoneo ${ }^{2}$. Además, raramente los coágulos en el árbol biliar pueden producir obstrucción de la vía biliar principal, como en nuestro caso ${ }^{9}$.

No está claro qué porcentaje de hemocolecistos es causado por malignidad, aunque se sabe que la neoplasia es causa del $6.2-6.8 \%$ de los casos descritos de hemobilia2-4. De estos, el $50 \%$ han surgido del hígado, el $24 \%$ de la vesícula biliar, el $24 \%$ de los conductos extrahepáticos y el $2 \%$ del páncreas ${ }^{10}$.

Se debería realizar colecistectomía de urgencia en caso de colecistitis gangrenosa asociada, para evitar complicaciones como la perforación. Si la sospecha es la presencia de un tumor, la indicación es el tratamiento quirúrgico.

Concluimos que el cáncer de vesícula biliar que se presenta como colecistitis aguda asociada a hemobilia y hemocolecisto es un cuadro clínico infrecuente, que se ha de tener en cuenta en el diagnóstico diferencial del dolor abdominal en el hipocondrio derecho, cuyo diagnóstico puede resultar inicialmente difícil, por lo que es importante conocer las diferentes formas de presentación.

\section{Responsabilidades éticas}

Protección de personas y animales. Los autores declaran que para esta investigación no se han realizado experimentos en seres humanos ni en animales.

Confidencialidad de los datos. Los autores declaran que han seguido los protocolos de su centro de trabajo sobre la publicación de datos de pacientes.

Derecho a la privacidad y consentimiento informado. Los autores han obtenido el consentimiento informado de los pacientes y/o sujetos referidos en el artículo. Este documento obra en poder del autor de correspondencia.

\section{Financiamiento}

Los autores declaran que no existen fuentes de financiamiento públicas o privadas en la realización del presente artículo.

\section{Conflicto de intereses}

Los autores declaran que no existe conflicto de intereses.

\section{Bibliografía}

1. Ruiz-Tovar J, Mingol F, Oller I, Santos J, Calpena R. Acute cholecystitis caused by hemocholecyst: unusual clinical manifestation of gallbladder cancer. Acta Gastroenterol Belg. 2013;76:57-8.

2. Díaz-Tobarra M, Garcés-Albir M, Moro-Valdezate D, Ortega-Serrano J. Hemocholecyst: a rare cause of acute abdomen. Rev Esp Enferm Dig. 2015;107:394-5.

3. Gomes AF, Fernandes S, Martins J, Coutinho J. Carcinoma of the gallbladder presenting as haemorrhagic cholecystitis. BMJ Case Reports. 2020;13:e232953.

4. Senthil Kumar AC, Balamurali S, Reshma S. Early gallbladder carcinoma with cholelithiasis: a rare case report. Int Surg J. 2017;4:2363-6.

5. Ku J, De La Rosa J, Kang J. Acute cholecystitis with a hemocholecyst as an unusual presentation of gallbladder cancer: report of a case. Surg Today. 2004;34:973-6.

6. Sharma A, Sharma KL, Gupta A, Yadav A, Kumar A. Gallbladder cancer epidemiology, pathogenesis and molecular genetics: recent update. World J Gastroenterol. 2017;23:3978-98.

7. Kim SH, Jung D, Ahn J-H, Kim KS. Differentiation between gallbladder cancer with acute cholecystitis: considerations for surgeons during emergency cholecystectomy, a cohort study. Int J Surg. 2017;45:1-7.

8. Kanthan R, Senger JL, Ahmed S, Kanthan SC. Gallbladder cancer in the $21^{\text {st }}$ century. J Oncol. 2015;2015:967472.

9. Fan Y, Wu SD, Kong J. Obstructive jaundice and melena caused by hemocholecyst: a case report. World J Gastroenterol. 2013;19:2126-8.

10. Ray S, Ghosh R, Khamrui S, Kumar Das A, Saha S, Kumar D. Haemocholecyst related to gallbladder malignancy. Trop Doct. 2020;50:243-5. 